\title{
Role of magnetic susceptibility-weighted imaging in characterization of cerebral microbleeds in acute ischemic stroke Egyptian obese patients
}

\author{
Ali Ahmed Abou Elmaaty ${ }^{1 *}$ and Carmen Ali Zarad ${ }^{2}$
}

\begin{abstract}
Objective: The study was done to investigate the role of susceptibility-weighted imaging (SWI) in characterization of cerebral microbleeds (CMBs) and incidence of CMBs in acute ischemic stroke obese patients.

Materials and methods: We recruited 120 acute ischemic stroke obese patients. All participants or their relatives gave written informed consent. MRI of the brain with SWI sequences was done for all patients.

Results: SWI showed $30 \%$ of the patients have CMBs. The total numbers of CMBs were statistically significant higher in small vessel diseases (SVDs) than large vessel diseases (LVDs) $(P=0.008)$. According to the severity scale, CMBs were more prominent in patients with grade 0 (70\%) followed by grade III (25.8\%) then grade II (4.2\%). BMI was independent risk factors for developing CMBs as shown in simple regression analysis $\left(x^{2}=6.008, P=0.002\right)$. SVDs and hypertension were statistically significant independent predictors and had higher odds to exhibit CMBs. Patients with SVDs had 5.3 times higher odds to exhibit CMBs $(P=0.002)$ while patients with hypertension had 3.9 times higher odds to exhibit CMBs $(P=0.032)$.

Conclusion: SWI is a gold standard MR imaging technique in diagnosis and characterization of CMBs with more prevalence especially in obese patients suffering from acute ischemia stroke due to SVDs. Hypertension was an independent risk factor for development of CMBs but IHD, use of antiplatelet medications, increased BMI, and dyslipidemia must be taken into consideration.
\end{abstract}

Keywords: Cerebral microbleeds, SWI, Ischemic stroke, Obesity, Vessel disease

\section{Introduction}

Obesity has a detrimental effect on health; it increases the risk of developing hypertension, cardiovascular disease, and cerebral stroke [1,2]. Obesity is considered a modifiable risk factor for both hemorrhagic and ischemic stroke in both sexes [3].

Several studies reported the linear relationship between body mass index (BMI) and ischemic stroke but not hemorrhagic stroke [4].

\footnotetext{
* Correspondence: carmenali042@gmail.com

${ }^{1}$ Badr University Hospital_-Helwan University, Helwan, Egypt

Full list of author information is available at the end of the article
}

Cerebral microbleeds (CMBs), white matter hyperintensities (WMH), and lacunes; all are common radiological imaging biomarkers of cerebral small vessel diseases (SVDs) [5].

CMBs have been observed in patients with ischemic and hemorrhagic stroke with frequency between 4 and $5 \%$ in healthy people, and can increase to $50-70 \%$ in patients with cerebrovascular disease [6].

Susceptibility-weighted imaging (SWI) is a newly developed MRI technique. SWI can differentiate the magnetic susceptibility effect of different components like blood products, deoxygenated blood, calcium, and iron. SWI employs a fully flow-compensated, high-resolution, 3D gradient-echo pulse using either separate or 
combined phase and magnitude images, in order to characterize brain tissue. Postprocessing of SW imaging includes combination of magnitude images with phase images [7].

SWI allows better detection of CMBs compared with 2D using a gradient-echo (GRE) pulse sequence. The high sensitivity detection rate of CMBs by SWI is due to the longer scan time as well as the high-spatial resolution 3D imaging [8].

In SWI, CMBs appear as small hypointense foci with size ranged from 2 to $10 \mathrm{~mm}$. CMBs occur as a result of focal accumulations of hemosiderin-containing macrophages that have a paramagnetic effect causing signal loss due to susceptibility effects [9].

In MRI, phase images can differentiate between calcification from CMBs as iron is a paramagnetic material while calcium is a diamagnetic material with opposing phase shifts so CMBs appear either low signal or high signal on phase image depending on the type of MR machine, whether the imager uses a left- or right-handed coordinate system [8].

CMBs are commonly asymptomatic but are considered a predictive factor of future recurrent ischemic and hemorrhagic stroke and considered to be clinically correlated with chronic hypertension, aging, and WMH $[10,11]$.

CMBs were classified into three groups according to their locations in SWI: lobar (cortical-subcortical white matter), deep (deep gray matter, basal ganglia, thalamus, the white matter of corpus callosum, internal, external and extreme capsule), and infra-tentorial (brain stem and cerebellum) [12, 13].

The distribution pattern of CMBs indicates different mechanisms, CMBs located in the deep white matter associated with hypertensive arteriopathy, whereas those in the lobe are more likely associated with cerebral amyloid angiopathy (CAA), with recent research shows that lobar CMBs are associated with changes in cognitive function $[14,15]$.

Many studies have shown that CMBs have not an important impact on hemorrhagic transformation only but they also consider as an independent risk factor for new ischemic stroke, transient ischemic attack, and stroke recurrence [16]. Therefore, the role of CMBs is important for the prevention and treatment of various strokes [17].

The aim of this study was to investigate the role of SWI in characterization of CMBs and incidence of CMBs in acute ischemic stroke obese patients.

\section{Patients and methods}

This is a cross sectional study that was approved by Research Ethics Committee (REC) for Human Subject and Animal Research at the Faculty of Medicine, Helwan University, Cairo, Egypt (serial13-2020). We recruited 120 patients admitted to the neurology department of Helwan University Hospitals from February to April 2020, these patients were obese and presented with acute ischemic stroke diagnosed by computed tomography (CT) brain, and all participants or their relatives gave written informed consent. The procedures we performed were in accordance with our protocol; brain MRI with SWI sequences was done for all patients.

We recruited obese patients of both sexes, $\geq 60$ years of age; patients that were before this age, showed mimic ischemic stroke diseases, refused consent, or who were contraindicated to brain MRI were excluded. All patients underwent full history taking and complete neurological examination stressing on medication drugs especially anticoagulants and antiplatelet therapy with high focus on vascular risk factors. Blood samples for laboratory investigation were obtained after overnight fasting and serum total cholesterol (TC), high-density lipoprotein (HDL-C), low-density lipoprotein cholesterol (LDL-C), triglyceride (TG), and fasting plasma glucose levels.

Acute ischemic stroke subtype SVDs and large vessel disease (LVDs) were diagnosed on the basis of cranial CT and MRI findings together with medical history, neurological signs and symptoms, and physical examination obtained at admission.

\section{Vascular risk factors}

Cerebral small vessel disease refers to a diverse range of clinical and neuroimaging findings resulting from pathological changes of various etiologies affecting the cerebral small vessels, particularly small veins, venules, capillaries, arterioles, and small arteries [18]. Cerebral large vessel disease is defined as disease affecting the proximal intracranial anterior and posterior circulation [19].

Hypertension was defined as self-reported hypertension, treatment with antihypertensive medication, a systolic blood pressure (SBP) $\geq 140 \mathrm{mmHg}$, and/or diastolic blood pressure (DBP) $\geq 90 \mathrm{~mm} \mathrm{Hg}$ [20]. Diabetes mellitus was defined as a fasting blood glucose level $126 \mathrm{mg} /$ $\mathrm{dL}$, a random blood glucose level $200 \mathrm{mg} / \mathrm{dL}$, or treatment with oral antidiabetic drugs or insulin [21].

Dyslipidemia was defined as a serum TC level $200 \mathrm{mg} /$ $\mathrm{dl}$, serum $\mathrm{TG} \geq 150 \mathrm{mg} / \mathrm{dL}$, HDL-C $\leq 40 \mathrm{mg} / \mathrm{dL}$ in males and $\leq 50 \mathrm{mg} / \mathrm{dL}$ in female, LDP-C $\geq 100 \mathrm{mg} / \mathrm{dL}$, or treatment with lipid-lowering drugs [22]. A current smoker was defined as a person who smoked at least 1 cigarette per day habitually or some days in the last 30 days preceding an interview [23]. Body mass index (BMI) calculated as weight in kilograms divided by height in meter squared and classified patients into overweight $\geq 25$ (preobese 25-29.99) and obese $\geq 30$ (grade I (30-34.99), grade II (35-34.99), and grade III $\geq 40$ ) [24].

\section{MRI and SWI sequence protocol}

All 120 patients performed conventional MRI with axial SWI images by $1.5-\mathrm{T}$ MR machine (Philips, Achieva 1.5 
T, Philips health care, Netherlands Holland); the following sequences were done: axial TSE T1-axial-weighted image (TR $400 \mathrm{~ms}$ TE $14 \mathrm{~ms}$ flip angle $90^{\circ}$ ), axial and coronal TSE T2-weighted image (WI, TR $2500 \mathrm{~ms}$ TE $88 \mathrm{~ms}$ flip angle, $90^{\circ}$ ), and axial and sagittal FLAIR FS (TR $9000 \mathrm{~ms}$ TE $127 \mathrm{~ms}$ ). Diffusion-sensitizing gradients were done, axial SWI (TR $78 \mathrm{~ms}$ TE $47 \mathrm{~ms}$ flip angle $15^{\circ}$ slice thickness $1.5 \mathrm{~mm}$ ) both the magnitude and phase images were done, and then post processing was done by combining the phase and magnitude images to create SWI and also created minimum intensity projection image (MIP)

\section{Image analysis}

CMBs were described as small round foci with hypointense signals that ranged from 2 to $10 \mathrm{~mm}$ in diameter on the SWI images.

Other areas of hypointense signal loss as vascular flow voids, areas of symmetric hypointensity, and areas in the basal ganglia, that represented calcification or nonhemorrhagic iron deposits, were excluded. Small foci of calcifications displayed hypointense signal loss in SWI images were excluded by phase images.

CMBs were classified into 3 types according to their location: lobar type that includes cortical or subcortical location (Fig. 1); deep type that included deep gray matter, thalamus, basal ganglia as well as white matter of the external, internal, extreme capsule, corpus callosum, cerebellum and brain stem (Fig. 2); and mixed type (Fig. 3) [11].

$\mathrm{CMBs}$ foci number was reported for each patient. According to the number of foci, the CMB severity was classified into 4 grades: in which grade $0=$ absent CMBs foci, grade $1=1 \mathrm{CMBs}$ foci lesion, grade $2=2$ to $5 \mathrm{CMBs}$ foci lesions, grade $3=$ more than $6 \mathrm{CMBs}$ foci lesions [25].

\section{Statistical analysis}

Data were entered and analyzed using IBM-SPSS software (IBM Corp. released 2017; IBM SPSS Statistics for Windows, Version 25.0. Armonk, NY: IBM Corp.). Qualitative data were expressed as frequency and percentage. Chi-square test or Fisher's exact test according to sample size of cells (expected counts) was used for comparing qualitative data. Chi-square test was used if sample size was $\geq 5$ units per cell while Fisher's exact test was used if sample size was $<5$ units in $\geq$ one cell. Quantitative data were initially tested for normality using Shapiro-Wilk's test with data being normally distributed if $P>0.050$. Presence of significant outliers (extreme values) was tested for by inspecting boxplots. Quantitative data were expressed as mean \pm standard deviation (SD) if normally distributed or median and interquartile range (IQR) if not. To compare quantitative data for two groups, Mann-Whitney $U$ test was used. Binary logistic regression analysis was used to predict the likelihood of occurrence of microbleeds. Crude odds ratio was initially calculated for each predictor and then a model was run to get the odds ratios of independent predictors. For any of the used tests, results were considered as statistically significant if $P$ value $\leq 0.050$.

\section{Results}

Qualitative and quantitative characteristics of the study cohort: Our study included 120 patients. Fifty-four patients were $(45 \%)$ males and 66 patients were $(55 \%)$ females; the participants aged from 60 to 88 years and their median age was 68 years [IQR was 64-75]. As regards BMI, 43 (35.8\%) patients were overweight, 77 (64.2\%) patients were obese [grade $\mathrm{I}=53$, grade $\mathrm{II}=18$, grade $\mathrm{III}=6$ patients]. The median BMI was 31.1(maximum was 45 and minimum was 25.8) and IQR 29.1-34.7.

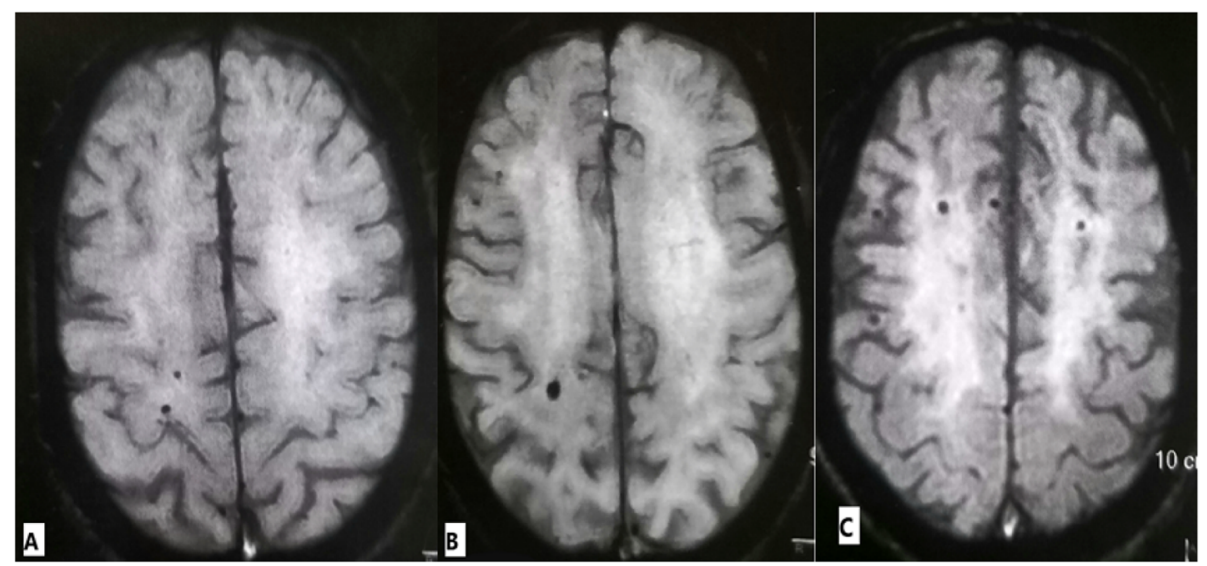

Fig. 1 Lobar type cerebral microbleeds. Axial brain SWI images (a-c) show multiple signal loss areas of cerebral microbleeds in the cortical gyri and subcortical white matter 


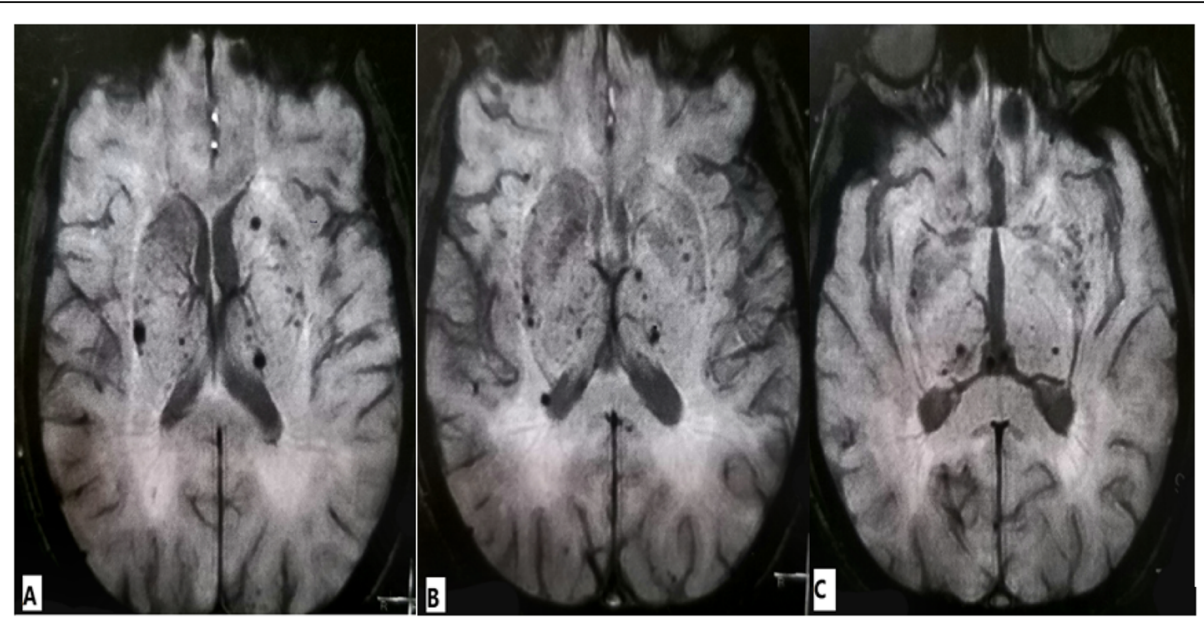

Fig. 2 Deep type cerebral microbleeds. Axial brain SWI images (a-c) show multiple signal loss areas of cerebral microbleeds in the periventricular deep white matter, basal ganglia, and both thalami

In our study, there were $62(51.7 \%)$ hypertensive patients, 48(40\%) diabetes mellitus (DM) patients, 53(44.2\%) IHD patients, 17 patients (14.2\%) were suffering from atrial fibrillation (AF), 25 patients $(20.8 \%)$ were smokers, 51patients $(42.5 \%)$ were on antiplatelet medications, 17 patients $(14.2 \%)$ were on anticoagulant medication, and 54 patients (45\%) suffered from dyslipidemia.

Brain MRI was done to all patients; 32 patients (26.7\%) suffered from SVDs and 88 patients $(73.3 \%)$ suffered from LVDs. SWI showed 36 patients (30\%) of cases suffered from CMBs (31 cortical, 35 deep, 36 mixed, and 18 infra-tentorial) (Table 1).

\section{Frequency of CMBs}

By SWI lobar type, CMBs ranged from 0 to 57; its median was 5 and IQR was 3 to 9 in SVDs, while in LVDs, CMBs ranged from 0 to 33, its median was 2 and IQR was 1-8. Lobar CMBs were statistically significantly higher in SVDs than LVDs $(P=0.066)$.

Deep type CMBs ranged from 0 to 80 , its median was 10 , and IQR was 7 to 23 in SVDs while it ranged from 1 to 48 , its median was 4 , and IQR was (2 to 8 ) in LVDs. Deep CMBs were statistically significantly higher in SVDs than LVDs $(P=0.008)$.

Mixed type CMBs ranged from 6 to 125, its median was 15, and IQR was 9 to 32 in SVDs, while LVD ranged from 2 to 81 , its median was 7 , and IQR was 3 to 14 . Mixed type CMBs were statistically significantly higher in SVDs than LVDs $(P=0.009)$.

Infra-tentorial type CMBs ranged from 0 to 18 , its median was 3, and IQR was 0 to 5 in SVDs, while it ranged from (0 to 3), its median was 0 , and IQR was (0 to 2$)$ in LVDs. Infra-tentorial CMBs were statistically significantly higher in SVDs than LVDs $(P=0.025)$.

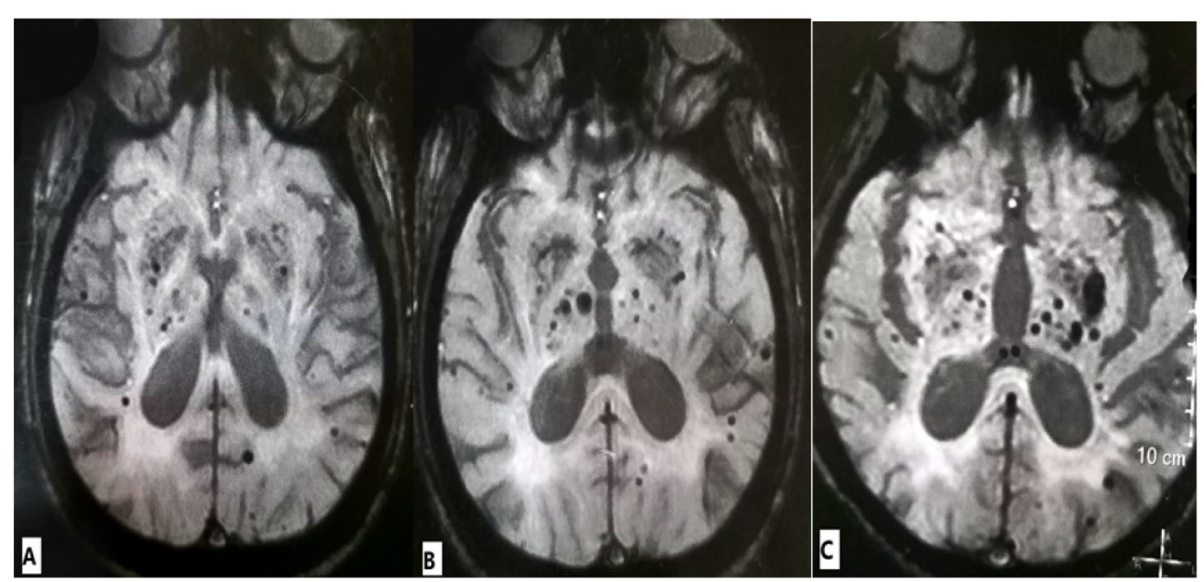

Fig. 3 Mixed type cerebral microbleeds. Axial brain SWI images (a-c) show bilateral multiple signal loss areas of cerebral microbleeds in the, basal ganglia, both thalami, deep periventricular and subcortical white matter, and cortical gyri 
Table 1 Characteristics of the study cohort $(n=120)$

\begin{tabular}{|c|c|}
\hline Qualitative data & Frequency (\%) \\
\hline \multicolumn{2}{|l|}{ Sex } \\
\hline Male & $54(45)$ \\
\hline Female & $66(55)$ \\
\hline \multicolumn{2}{|l|}{ BMI category } \\
\hline Overweight & $43(35.8)$ \\
\hline Obese & $77(64.2)$ \\
\hline Grade I & 53 \\
\hline Grade II & 18 \\
\hline Grade III & 06 \\
\hline Presence of hypertension & $62(51.7)$ \\
\hline Presence of diabetes & $48(40)$ \\
\hline Presence of dyslipidemia & $54(45)$ \\
\hline Presence of IHD & $53(44.2)$ \\
\hline Presence of AF & $17(14.2)$ \\
\hline A current smoker & $25(20.8)$ \\
\hline On antiplatelet medications & $51(42.5)$ \\
\hline On anticoagulant medications & $17(14.2)$ \\
\hline \multicolumn{2}{|l|}{ Brain MRI } \\
\hline SVDs & $32(26.7)$ \\
\hline LVDs & $88(73.3)$ \\
\hline \multicolumn{2}{|l|}{ SWI } \\
\hline $\mathrm{CMBs}$ & $36(30)$ \\
\hline lobar & 31 \\
\hline Deep & 35 \\
\hline Mixed & 36 \\
\hline Infra-tentorial & 18 \\
\hline Quantitative data & Median (IQR), minimum, maximum \\
\hline Age (years) & $68(64-75), 60,88$ \\
\hline BMI $\left(\mathrm{kg} / \mathrm{m}^{2}\right)$ & $31.1(29.1-34.7), 25.8,45$ \\
\hline Serum TC (mg/dL) & 186 (152-249), 103, 275 \\
\hline Serum TG (mg/dL) & $139.5(109.3-162), 49,280$ \\
\hline Serum LDL-C (mg/dL) & $44(39.3-56), 23,106$ \\
\hline Serum HDL-C (mg/dL) & 97 (69-151.5), 32.8, 184 \\
\hline
\end{tabular}

IHD ischemic heart disease, $A F$ atrial fibrillation, MRI magnetic resonance imaging, SWI susceptibility-weighted imaging, BMI body mass index, SVDs small vessel disease, $L V D s$ large vessel disease, $C M B s$ cerebral microbleeds, $T C$ total cholesterol, $T G$ triglycerides, $L D L$ low-density lipoprotein, $H D L$ high-density lipoprotein

In comparing total numbers of CMBs in SVD and LVD, there were statistically significant higher number of CMBs in SVDs than LVDs $(P=0.008)$ (Table 2).

\section{Severity of CMBs}

According to severity of CMBs by SWI, there were 84 patients $(70 \%)$ with grade 0,5 patients $(4.2 \%)$ grade II, and 30 patients $(25.8 \%)$ grade III and there was no patient in grade $\mathrm{I}$.

\section{Univariate analysis of predictors of CMBs}

Simple logistic regression test was run to ascertain the effects of sex, BMI $\left(\mathrm{kg} / \mathrm{m}^{2}\right)$, hypertension, DM, dyslipidemia, AF, IHD, smoking history, antiplatelet drugs, anticoagulant therapy, MRI, serum TC $(\mathrm{mg} / \mathrm{dl})$, serum TG (mg/dl), LDL-C (mg/dl), and serum HDL-C (mg/dl) on the likelihood that participants CMBs. Of all univariate predictors of CMBs, the simple logistic regression model was statistically significant by comparing obese versus overweight $\left(x^{2}=6.008, P=0.002\right)$ and has 3.107 higher odds to exhibit CMBs. An increase in BMI category by one class has 2.223 higher odds to exhibit CMBs. The simple logistic regression model was statistically significant as univariate predictors of CMBs for dyslipidemia versus non-dyslipidemia $\left(x^{2}=7.414, P=0.008\right)$ has 3.025 odds to exhibit CMBs; also the simple logistic regression model was statistically significant as univariate predictors of $\mathrm{CMBs}$ for IHD, antiplatelet drug therapy $(P$ $=0.016$ and $P=0.023)$, respectively. The simple logistic regression model for brain MRI showed SVDs versus LVDs was the strongest univariate predictors of CMBs $\left(x^{2}=31.202, P<0.001\right)$ and had 11.629 higher odds to exhibit CMBs followed by hypertension versus normotensive patients $\left(x^{2}=20.652, P<0.001\right)$ and had 8.125 higher odds to exhibit CMBs as in Table 3

\section{Predictors of the likelihood of occurrence of CMBs}

A binomial logistic regression was run to ascertain the effects of presence of SVDs on MRI, presence of hypertension, BMI $(\mathrm{kg} / \mathrm{m} 2)$, serum TC $(\mathrm{mg} / \mathrm{dl})$, LDL-C $(\mathrm{mg} /$ $\mathrm{dl}$ ), presence of IHD, and use of antiplatelet medications on the likelihood that participants have microbleeds. There were 5 studentized residuals with values of 2.167 , $2.167,2.477,2.002$, and 2.272 SD which were kept in the analysis. The logistic regression model was statistically significant, $X^{2}(7)=39.376, P<0.001$. The model explained $39.7 \%$ (Nagelkerke $R^{2}$ ) of the variance in microbleeds and correctly classified $82.5 \%$ of cases. Sensitivity was $61.1 \%$, specificity was $91.7 \%$, positive predictive value was $75.9 \%$, and negative predictive value was $84.6 \%$. Of the eight predictor variables, only presence of small vessel disease on MRI and presence of hypertension were statistically significant independent predictors. Patients with small vessel disease on MRI had 5.3 times higher odds to exhibit microbleeds $(P=0.002)$ and patients with hypertension had 3.9 times higher odds to exhibit microbleeds $(P=0.032)$ as in Table 4 .

\section{Discussion}

In this study, SWI showed 36 patients suffered from CMBs (31 cortical, 35 deep, 36 mixed and 18 infratentorial); according to that, the prevalence of CMBs in this study was $30 \%$ (68.8\% in SVDs \& $15.9 \%$ in LVDs) with high statistically significantly difference in SVDs $\left(\chi^{2}\right.$ 
Table 2 Frequency and location of CMBs

\begin{tabular}{|c|c|c|c|c|c|c|}
\hline \multirow[t]{2}{*}{ Location } & \multicolumn{2}{|l|}{$\operatorname{SVD}(n=32)$} & \multicolumn{2}{|l|}{ LVDs $(n=88)$} & \multirow{2}{*}{$\begin{array}{l} \\
\text { value }\end{array}$} & \multirow{2}{*}{$\begin{array}{l}P \\
\text { value }\end{array}$} \\
\hline & Median (IQR) & Range & Median (IQR) & Range & & \\
\hline Lobar & $5(3-9)$ & $0-57$ & $2(1-8)$ & $0-33$ & -1.845 & 0.066 \\
\hline Deep & $10(7-23)$ & $0-80$ & $4(2-8)$ & $1-48$ & -2.621 & 0.008 \\
\hline Mixed & $15(9-32)$ & $6-125$ & $7(3-14)$ & $2-81$ & -2.584 & 0.009 \\
\hline Infra-tentorial & $3(0-5)$ & $0-18$ & $0(0-2)$ & $0-3$ & -2.399 & 0.025 \\
\hline Total & $16(12-34)$ & $7-143$ & $8(3-16)$ & $2-81$ & -2.618 & 0.008 \\
\hline
\end{tabular}

$P$ value: Mann-Whitney $U$ test. SVDs small vessel diseases, $L V D$ s large vessel diseases

$=31.201, P<0.001)$; while Potigumjon and his colleagues reported that the prevalence of CMBs in ischemic stroke patients was $20 \%$, Kwa and his colleagues found that the incidence of CMBs in ischemic stroke patient was $6 \%[26,27]$.

In our study, the number of CMBs was statistically significantly higher in SVDs versus LVDs in comparing location: deep, mixed (lobar and deep), infra-tentorial, and total number in both groups $(P=0.008, P=0.009, P=$ $0.025, P=0.008)$, respectively, but not in lobar location $(P=0.066)$.

In this study, we observed the majority of the cases presented with CMBs were mixed, deep, lobar then infra-tentorial location; this was inconsistent with Pantoni who reported that CMBs were mainly observed in lobar brain regions [28].

As regards $\mathrm{MBI}, \mathrm{CMBs}$ were higher significantly difference in the obese group when compared with the overweight group which had 3.107 higher odds to exhibit CMB $\left(\chi^{2}=6.008, P=0.017\right)$, furthermore, an increase in BMI category by one grade has 2.223 higher odds to exhibit $\mathrm{CMB}$, (grade II $=4.446$, grade $\mathrm{III}=6.669)(P=$ 0.002 ). This is consistent with Mitaki and his colleagues; they found that increased BMI was associated with increased incidence of deep and infra-tentorial CMBs; however, the positive association between incidence of CMBs and BMI is still controversial [11].

In our study hypertension was strong independent risk factor in development of CMBs $\left(\chi^{2}=20.652, P<0.001\right)$; this was consistent with Vernooij et al. [10] as they reported CMBs were independently associated with higher systolic blood pressure and inconsistent with Mitaki and his colleagues who found that the hypertension were not associated with increased incidence of CMBs [11].

In this study, we found that CMBs were statistically significantly higher in dyslipidemic patients $\left(\chi^{2}=7.414\right.$, $P=0.008)$. Serum TC and serum LDL-C were significantly associated with increased incidence of CMBs $(P=$ 0.002 and $P=0.004)$, respectively, with no association with serum TG and serum HDL-C. This is inconsistent with Mitaki et al. [11] that dyslipidemia was not associated with increased incidence of CMBs.
In this study, we founded that patients who suffered from IHD and received antiplatelet drugs were more prone to develop CMBs with higher statistically significant difference when compared with nonusers $\left(\chi^{2}=\right.$ 5.988, $P=0.016$ ); this is consistent with Huo and his colleagues and also with Vernooij and his colleagues that the prevalence of CMBs were higher in patients treated with antiplatelet drugs used in the primary and secondary prevention of thrombotic cardiovascular disease, including acute coronary syndrome compared with nonusers $[29,30]$.

We founded in our research that the prevalence of $\mathrm{CMB}$ was higher in patients who received antiplatelet with a statistically significant difference when compared with nonuser (58.3\%), $P=0.023$. while Lihong and his colleagues reported that CMBs occurred in $40.0 \%$ of patients taking aspirin for at least 1 year and $30.7 \%$ of patients taking clopidogrel for at least 1 year [31].

In our study, we founded that patients with SVDs on brain MRI were statistically significantly higher when compared with LVDs to develop CMBs $\left(\chi^{2}=31.201, P<\right.$ 0.001).

In this study, we did not find any statistically significant difference as regards sex (female and male), diabetic and non-diabetic, and AF and non AF patients; patients received anticoagulant therapy, serum TG, and serum HDL-C in prevalence of CMBs. Mitaki et al. [11] found that elevated fasting glucose was not associated with increased incidence of CMBs, inconsistent with Saito and his colleagues who reported that the incidence of CMBs in patients with AF is significantly higher than that in patients without AF [32].

In our research, we found that a binomial logistic regression to ascertain the effects of presence of SVDs on MRI, presence of hypertension, BMI $(\mathrm{kg} / \mathrm{m} 2)$, serum TC (mg/dl), LDL-C (mg/dl), presence of IHD, and use of antiplatelet medications on the likelihood that participants had microbleeds. Of the seven predictor variables, only the presence of small vessel disease on MRI and presence of hypertension were statistically significant independent predictors to develop CMBs. Patients with SVDs on SWI had 5.3 times higher odds to exhibit microbleeds $(P=$ 
Table 3 Univariate analysis of predictors of CMBs

\begin{tabular}{|c|c|c|c|c|c|c|}
\hline \multirow[t]{2}{*}{ Predictor } & \multirow{2}{*}{$\begin{array}{l}\text { No microbleeds } \\
(n=84)\end{array}$} & \multirow{2}{*}{$\begin{array}{l}\text { Microbleeds ( } n= \\
36 \text { ) }\end{array}$} & \multicolumn{2}{|c|}{ Test of significance } & \multicolumn{2}{|c|}{ Simple logistic regression } \\
\hline & & & Statistic & P1 & Crude odds ratio & P2 \\
\hline \multicolumn{7}{|l|}{ Sex } \\
\hline Female & $45(53.6 \%)$ & $21(58.3 \%)$ & $x^{2}=0.231$ & 0.631 & $R$ & 0.631 \\
\hline Male & 39 (46.4\%) & $15(41.7 \%)$ & & & 0.824 & \\
\hline \multicolumn{7}{|l|}{ BMl } \\
\hline Overweight & $36(42.9 \%)$ & $7(19.4 \%)$ & $x^{2}=6.008$ & 0.014 & $R$ & 0.017 \\
\hline Obese & $48(57.1 \%)$ & 29 (80.6\%) & & & 3.107 & \\
\hline Grade I & $37(44.0 \%)$ & $16(44.4 \%)$ & FET & 0.010 & 2.223 & 0.002 \\
\hline Grade II & 09 (10.7\%) & 09 (25.0\%) & & & & \\
\hline Grade III & $02(2.4 \%)$ & $04(11.1 \%)$ & & & & \\
\hline \multicolumn{7}{|l|}{ Hypertension } \\
\hline No & $52(61.9 \%)$ & $6(16.7 \%)$ & $x^{2}=20.652$ & $<0.001$ & $R$ & $<0.001$ \\
\hline Yes & $32(38.1 \%)$ & $30(83.3 \%)$ & & & 8.125 & \\
\hline \multicolumn{7}{|l|}{ DM } \\
\hline No & 49 (58.3\%) & $23(63.9 \%)$ & $x^{2}=0.324$ & 0.569 & $R$ & 0.570 \\
\hline Yes & $35(41.7 \%)$ & $13(36.1 \%)$ & & & 0.791 & \\
\hline \multicolumn{7}{|l|}{ Dyslipidemia } \\
\hline No & $53(63.1 \%)$ & $13(36.1 \%)$ & $x^{2}=7.414$ & 0.006 & $R$ & 0.008 \\
\hline Yes & 31 (36.9\%) & $23(63.9 \%)$ & & & 3.025 & \\
\hline \multicolumn{7}{|l|}{ AF } \\
\hline No & $69(82.1 \%)$ & $34(94.4 \%)$ & $x^{2}=3.136$ & 0.077 & $R$ & 0.094 \\
\hline Yes & 15 (17.9\%) & $2(5.6 \%)$ & & & 0.271 & \\
\hline \multicolumn{7}{|l|}{ IHD } \\
\hline No & $53(63.1 \%)$ & $14(38.9 \%)$ & $x^{2}=5.988$ & 0.014 & $R$ & 0.016 \\
\hline Yes & 31 (36.9\%) & $22(61.1 \%)$ & & & 2.687 & \\
\hline \multicolumn{7}{|l|}{ On antiplatelet drugs } \\
\hline No & $54(64.3 \%)$ & $15(41.7 \%)$ & $x^{2}=5.276$ & 0.022 & $R$ & 0.023 \\
\hline Yes & $30(35.7 \%)$ & $21(58.3 \%)$ & & & 2.520 & \\
\hline \multicolumn{7}{|l|}{ On anticoagulant drugs } \\
\hline No & $69(82.1 \%)$ & $34(94.4 \%)$ & $x^{2}=3.136$ & 0.077 & $R$ & 0.094 \\
\hline Yes & 15 (17.9\%) & $2(5.6 \%)$ & & & 0.271 & \\
\hline \multicolumn{7}{|l|}{ Brain MRI } \\
\hline LVDs & $74(88.1 \%)$ & $14(38.9 \%)$ & $x^{2}=31.201$ & $<0.001$ & $R$ & $<0.001$ \\
\hline SVDs & $10(11.9 \%)$ & $22(61.1 \%)$ & & & 11.629 & \\
\hline Age (years) & $68.5(63-75)$ & $68(65.3-73.8)$ & $Z=-0.077$ & 0.938 & 0.988 & 0.689 \\
\hline $\mathrm{BMI}\left(\mathrm{kg} / \mathrm{m}^{2}\right)$ & $30.6(28.7-33.97)$ & $33.5(30.9-37.2)$ & $Z=-3.196$ & 0.001 & 1.195 & 0.001 \\
\hline Serum TC (mg/dl) & $179.5(144.5-227)$ & $233(175-251)$ & $Z=-3.064$ & 0.002 & 1.013 & 0.002 \\
\hline Serum TG (mg/dl) & 129 (109-161) & $146.5(110.5-169.5)$ & $Z=-0.465$ & 0.642 & 1.000 & 0.918 \\
\hline Serum HDL-C (mg/dl) & $43(35.3-51.8)$ & 44.5 (41.3-59) & $Z=-1.750$ & 0.080 & 1.029 & 0.037 \\
\hline Serum LDL-C (mg/dl) & $96(68-137.8)$ & $141(69-175.8)$ & $Z=-3.112$ & 0.002 & 1.014 & 0.004 \\
\hline
\end{tabular}

$R$ reference category, $C O R$ crude odds ratio, $P 1, P 2$ simple logistic regression, $D M$ diabetes mellitus, $A F$ atrial fibrillation, $I H D$ ischemic heart disease, $L V D s$ large vessel diseases, SVDs small vessel diseases, BMI body mass index, TC total cholesterol, $T G$ triglyceride, $H D L-C$ high-density lipoprotein-C, $L D I-C$ low-density lipoprotein-C 
Table 4 Predictors of the likelihood of occurrence of CMBs

\begin{tabular}{|c|c|c|c|c|c|}
\hline Predictor & $B$ & SE & Wald & $P$ value & OR $(95 \% \mathrm{Cl})$ \\
\hline Brain MRI & 1.669 & 0.551 & 9.176 & 0.002 & $5.307(1.802-15.627)$ \\
\hline \multicolumn{6}{|l|}{ LVDs } \\
\hline \multicolumn{6}{|l|}{ SVDs } \\
\hline Hypertension & 1.363 & 0.637 & 4.586 & 0.032 & $3.909(1.123-13.612)$ \\
\hline \multicolumn{6}{|l|}{ No } \\
\hline \multicolumn{6}{|l|}{ Yes } \\
\hline $\mathrm{BMI}\left(\mathrm{kg} / \mathrm{m}^{2}\right)$ & -0.015 & 0.080 & 0.037 & 0.848 & $0.985(0.842-1.152)$ \\
\hline Serum TC (mg/dl) & 0.004 & 0.012 & 0.082 & 0.775 & $1.004(0.980-1.028)$ \\
\hline Serum LDL-C (mg/dl) & 0.001 & 0.014 & 0.003 & 0.958 & $1.001(0.874-1.028)$ \\
\hline IHD & 0.386 & 1.586 & 0.059 & 0.808 & $1.471(0.266-32.910)$ \\
\hline \multicolumn{6}{|l|}{ No } \\
\hline \multicolumn{6}{|l|}{ Yes } \\
\hline On antiplatelet medications & 0.426 & 1.597 & 0.071 & 0.790 & $1.531(0.067-35.021)$ \\
\hline \multicolumn{6}{|l|}{ No } \\
\hline Yes & & & & & \\
\hline
\end{tabular}

$B$ binary logistic regression coefficient, $S E$ standard error, $O R$ odds ratio, $C I$ confidence interval, $L V D s$ large vessel diseases, SVDs small vessel diseases, $B M I$ body mass index, TC total cholesterol, $L D I-C$ low-density lipoprotein, IHD ischemic heart disease

0.002), and patients with hypertension had 3.9 times higher odds to exhibit microbleeds $(P=0.032)$

\section{Conclusion}

SWI is a gold standard MR imaging technique in diagnosis and characterization of CMBs with more prevalence especially in obese patients suffering from acute ischemia stroke due to small vessel disease. Hypertension is an independent risk factor for the development of CMBs but IHD, use of antiplatelet medications, increased BMI, and dyslipidemia must be taken into consideration.

\section{Abbreviation}

AF: Atrial fibrillation; BMI: Body mass index; BP: Blood pressure; CAA: Cerebral amyloid angiopathy; CMBs: Cerebral microbleeds; CT: Computed tomography; DBP: Diastolic blood pressure; DM: Diabetes mellitus; GRE: Gradient recall echo; HDL: High-density lipoprotein; IHD: Ischemic heart disease; IQR: Interquartile range; LDL: Low-density lipoprotein; LVDs: Large vessel diseases; MRI: Magnetic resonance imaging; SBP: Systolic blood pressure; SD: Standard deviation; SVDs: Small vessel diseases;

SWI: Susceptibility-weighted imaging; TC: Total cholesterol; TG: Triglyceride; WI: Weighted imaging; WMD: White matter disease

\section{Acknowledgements}

The authors are grateful to all patients and control subjects for their willingness to participate in this study.

\section{Authors' contributions}

AAM and CAZ carried out the work. AAM performed the procedure, did the statistical analysis, and was responsible for collecting the scientific data and writing the initial draft of the manuscript. CAZ designed the protocol shared in collecting the scientific data. CAZ interpreted the radiology of all patients. Both authors read and approved the final version to be published.

\section{Funding}

There is no source of funding for the research.

\section{Availability of data and materials}

The data supporting the results of this article are included within the article.

\section{Ethics approval and consent to participate}

The authors obtained permission to conduct this study that was approved by Research Ethics Committee (REC) for Human Subject and animal Research at the Faculty of Medicine, Helwan University, Cairo, Egypt (serial 13-2020). All participants gave written informed consent. The procedures followed were in accordance with our protocol. We recruited 120 patients admitted to the neurology department of Helwan University Hospitals from February to April 2020.

\section{Consent for publication}

Not applicable.

\section{Competing interests}

The authors declare that they have no competing interests.

\section{Author details}

${ }^{1}$ Badr University Hospital_-Helwan University, Helwan, Egypt. ${ }^{2}$ Port Said University, Port Said, Egypt.

Received: 1 May 2020 Accepted: 22 June 2020

Published online: 08 July 2020

\section{References}

1. Flegal KM, Kit BK, Orpana H, Graubard BI. Association of all- cause mortality with overweight and obesity using standard body mass index categories: a systematic review and meta-analysis. JAMA. 2013;309:71-82.

2. Strazzullo P, D’Elia L, Cairella G, Garbagnati F, Cappuccio FP, Scalfi L. Excess body weight and incidence of stroke: Meta-analysis of prospective studies with 2 million participants. Stroke. 2010;41:e418-26.

3. Ni Mhurchu C, Rodgers A, Pan WH, Gu DF, Woodward M. Body mass index and cardiovascular disease in the Asia-Pacific Region: an overview of 33 cohorts involving 310000 participants. Int J Epidemiol. 2004;33:751-8.

4. Park JW, Lee S-Y, Kim SY, Choe H, Jee SH. BMI and stroke risk in Korean women. Obesity. 2008;16:396-401.

5. Wardlaw JM, Smith EE, Biessels GJ, Cordonnier C, Fazekas F, Frayne R. Neuroimaging standards for research into small vessel disease and its contribution to ageing and neurodegeneration. Lancet Neurol. 2013;12:822-38. 
6. Greenberg SM, Vernooij MW, Cordonnier C, Viswanathan A, Salman R, Warach AS, et al. Cerebral microbleeds: a guide to detection and interpretation. Lancet Neurol. 2009:8:165-74.

7. Offenbacher H, Fazekas F, Schmidt R, Koch M, Fazekas G, Kapeller P. MR of cerebral abnormalities concomitant with primary intracerebral hematomas. AJNR Am. J. Neuroradiol. 1996;17:573-8.

8. Han F, Zhai F, Wang Q, Zhou L, Ni J, Yao M, et al. Prevalence and risk factors of cerebral small vessel disease in a Chinese population-based sample. J Stroke. 2018;20(2):239-46.

9. Thijs V, Lemmens R, Schoofs C, Gorner A, Van Damme P, Schrooten M, et al. Microbleeds and the risk of recurrent stroke. Stroke. 2010;41(9):2005-9.

10. Vernooij MW, van der Lugt A, Ikram MA, Wielopolski PA, Niessen WJ, Hofman A, et al. Prevalence and risk factors of cerebral microbleeds: The Rotterdam Scan Study. Neurology. 2008;70:1208-14.

11. Mitaki S, Takayoshi H, Nakagawa T, Nagai A, Oguro H, Yamaguchi S. Metabolic syndrome is associated with incidence of deep cerebral microbleeds. PLoS One. 2018;13(3):e0194182

12. Chung CP, Chou KH, Chen WT, Liu LK, Lee WJ, Chen LK, et al. Strictly lobar cerebral microbleeds are associated with cognitive impairment. Stroke. 2016;47:2497-502.

13. Shams S, Granberg T, Martola J, Charidimou A, Li X, Shams M, et al. Cerebral microbleeds topography and cerebrospinal fluid biomarkers in cognitive impairment. J Cereb Blood Flow Metab. 2017;37:1006-13.

14. Boeckh-Behrens T, Lutz J, Lummel N, Burke M, Wesemann T, Schöpf V, et al. Susceptibility-weighted angiography (SWAN) of cerebral veins and arteries compared to TOF-MRA. Eur J Radiol. 2012;81:1238-45.

15. Shams S, Martola J, Cavallin L, Granberg T, Shams M, Aspelin P, et al. SWI or T2*: which MRI sequence to use in the detection of cerebral microbleeds? The Karolinska Imaging Dementia Study. Am J Neuroradiol. 2015;36(6):108995.

16. Soo YO, Siu DY, Abrigo J, Yu S, Ng N, Ahuja AT, et al. Risk of intracerebral hemorrhage in patients with cerebral microbleeds undergoing endovascular intervention. Stroke. 2012;43:1532-6.

17. Charidimou A, Kakar P, Fox Z, Werring DJ. Cerebral microbleeds and recurrent stroke risk: systematic review and meta-analysis of prospective ischemic stroke and transient ischemic attack cohorts. Stroke. 2013;44:9951001.

18. Pantoni L, Gorelick PB. Cerebral small vessel disease. First ed. Cambridge: Cambridge University Press; 2014.

19. Rennert RC, Wali AR, Steinberg JA, Santiago-Dieppa DR, Olson SE, Pannell JS, Khalessi AA. Neurosurgery. 2019;85(Suppl 1):S4-8.

20. SPRINT Research Group, Wright JT Jr, Williamson JD, Whelton PK, Snyder JK, et al. A randomized trial of intensive versus standard blood-pressure control. N Engl J Med. 2015;373:2103-16.

21. Association AD. Diagnosis and classification of diabetes mellitus. Diabetes Care. 2014;37(Suppl, 1):S81-90.

22. Stone NJ, Robinson JG, Lichtenstein AH, Bairey Merz CN, Blum CB, Eckel RH, et al. 2013 ACC/AHA guideline on the treatment of blood cholesterol to reduce atherosclerotic cardiovascular risk in adults: a report of the American College of Cardiology/American Heart Association task force on practice guidelines. J Am Coll Cardiol. 2014;63:2889-934.

23. World Health Organization. WHO Report on the Global Tobacco Epidemic, 2019. Geneva: World Health Organization; 2019. Available from: https://apps who.int/iris/bitstream/handle/10665/326043/9789241516204-eng.pdf.

24. Consultation WHO. Appropriate body-mass index for Asian populations and its implications for policy and intervention strategies. Lancet. 2004;363:15763.

25. Gao Z, Wang W, Wang Z, Zhao X, Shang Y, et al. Cerebral microbleeds are associated with deep white matter hyperintensities, but only in hypertensive patients. PLoS ONE. 2014;9(3):e91637. https://doi.org/10.1371/ journal.pone.0091637.

26. Potigumjon A, Watcharakorn A, Dharmasaroja PA. Prevalence of cerebral microbleeds in Thai patients with ischemic stroke. J Neurosci Rural Pract. 2017:8:216-20

27. Kwa VI, Franke CL. Verbeeten B jr, Stam J. Silent intracerebral microhemorrahage in patients with ischemic stroke. Amsterdam vascular Medicine group. Ann Neurol. 1998;44:362-7.

28. Pantoni L. Cerebral small vessel disease: from pathogenesis and clinical characteristics to therapeutic challenges. Lancet Neurol. 2010;9:689-701.

29. Huo Y, Zhang Y, Han Y, Yan H, Ge J. Cardiovascular diseases in China: the blue book myocardial infarction. Cardiol Plus. 2017;2:39-54.
30. Vernooij MW, Haag MD, van der Lugt A, Hofman A, Krestin GP, Stricker BH, et al. Use of antithrombotic drugs and the presence of cerebral microbleeds: The Rotterdam Scan Study. Arch Neurol. 2009;66:6.714-20.

31. Ge L, Ouyang X, Ban C, Yu H, Wu Q, Wu H, et al. Cerebral microbleeds in patients with ischemic cerebrovascular disease taking aspirin or clopidogrel. Medicine (Baltimore). 2019;98(9):e14685.

32. Saito T, Kawamura Y, Tanabe Y, Asanome A, Takahashi K, Sawada J, et al. Cerebral microbleeds and asymptomatic cerebral infarctions in patients with atrial fibrillation. J Stroke Cerebrovasc Dis. 2014;23:1616-22.

\section{Publisher's Note}

Springer Nature remains neutral with regard to jurisdictional claims in published maps and institutional affiliations.

\section{Submit your manuscript to a SpringerOpen ${ }^{\circ}$ journal and benefit from:}

- Convenient online submission

- Rigorous peer review

- Open access: articles freely available online

- High visibility within the field

- Retaining the copyright to your article

Submit your next manuscript at $\boldsymbol{\nabla}$ springeropen.com 